\title{
Erratum to: Mitochondrial phospholipids: role in mitochondrial function
}

\author{
Edgard M. Mejia • Grant M. Hatch
}

Published online: 17 March 2015

(C) Springer Science+Business Media New York 2015

Erratum to: J Bioenerg Biomembr (2015)

DOI 10.1007/s10863-015-9601-4

The original version of this article unfortunately contains errors in the orientation of the lipid bilayers of Fig. 1. The updated figure is available here.

The online version of the original article can be found at http://dx.doi.org/ 10.1007/s10863-015-9601-4.

E. M. Mejia • G. M. Hatch $(\bowtie)$

Department of Pharmacology and Therapeutics, University of

Manitoba, Winnipeg, MB, Canada

e-mail: ghatch@chrim.ca

G. M. Hatch

Biochemistry and Medical Genetics, Center for Research and Treatment of Atherosclersosis, University of Manitoba and DREAM

Theme Children's Hospital Research Institute Manitoba,

Winnipeg, MB, Canada 


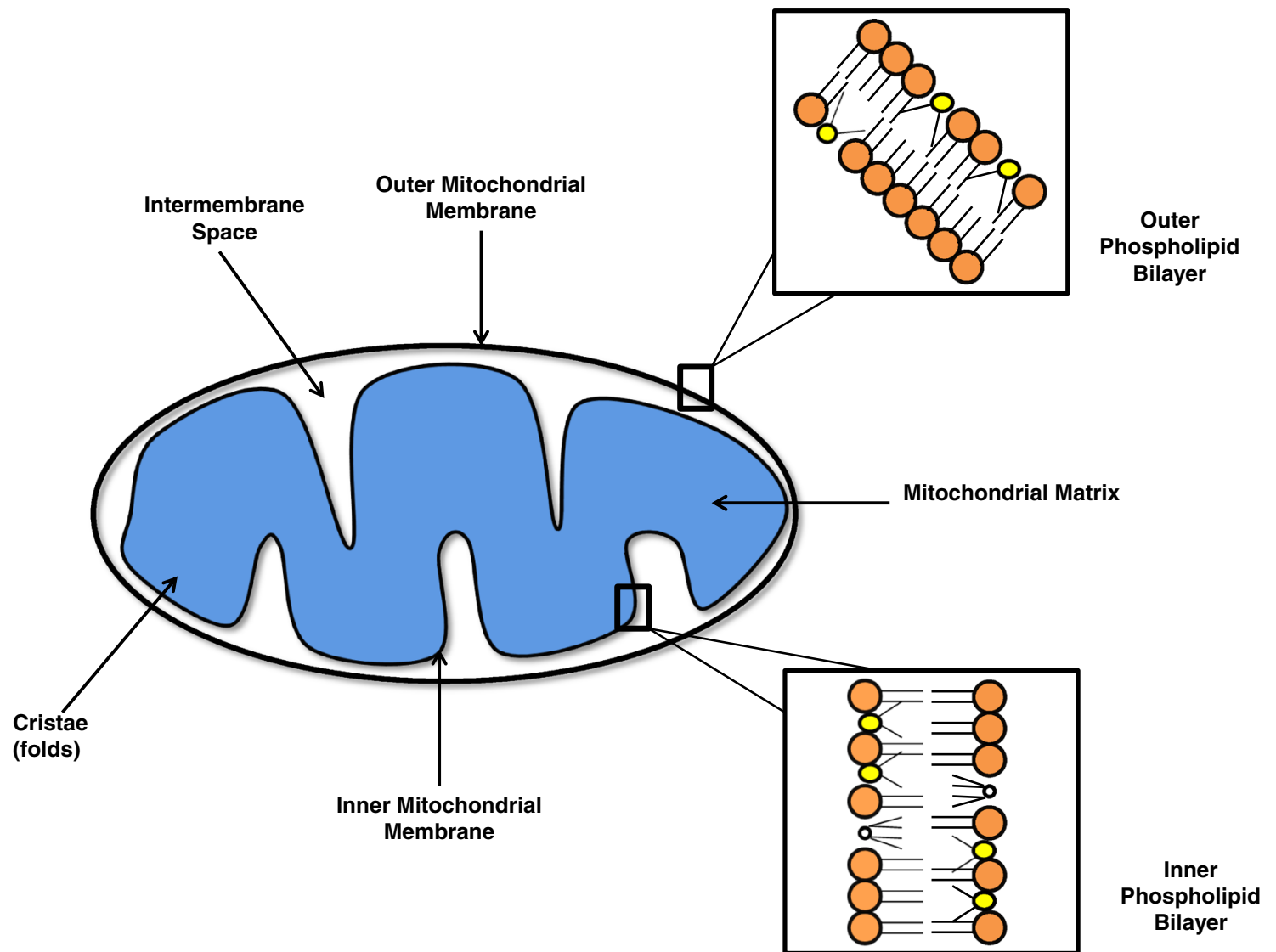

Fig. 1 The outer mitochondrial membrane (OMM) and inner mitochondrial membrane (IMM) contain structural phospholipids such as phosphatidylcholine (PC), phosphatidylethanolamine (PE) and cardiolipin (CL). Phospholipid abundance varies between the OMM and IMM. PC and PE make up the majority of phospholipids, and PE and $\mathrm{CL}$ are more abundant in the IMM compared to the OMM. The tubular formation of PC allows for the formation of planar bilayers in both the OMM and IMM. Non-bilayer forming phospholipids such as PE and CL have a smaller hydrophilic head group compared to their hydrophobic acyl groups, thus favouring hexagonal phase formations. This introduces tension into mitochondrial membranes which allows the incorporation of proteins and can also contribute to the negative curvature of the membrane thereby facilitating specific mitochondrial functions such as fusion and/or fission. [Orange $=\mathrm{PC}$, yellow $=\mathrm{PE}$, white $=\mathrm{CL}$ ] 